\title{
Experiência de Uso de Caixas de Ovos no Apoio ao Ensino de Vetores e Matrizes
}

\author{
Rita C. G. Berardi ${ }^{1}$, Silvia A. Bim ${ }^{1}$, Regiane Macuch $^{2}$, Leticia Fleig Dal Forno² \\ ${ }^{1}$ Departamento Acadêmico de Informática - Universidade Tecnológica Federal do \\ Paraná (UTFPR) \\ Curitiba - PR - Brasil \\ ${ }^{2}$ Centro Universitário de Maringá (UNICESUMAR)- Bolsista Produtividade ICETI \\ Maringá - PR - Brasil \\ \{ritaberardi, sabim\} @utfpr.edu.br, \\ \{leticia.forno, regiane.macuch\} @unicesumar.edu.br
}

\begin{abstract}
The programming discipline is embedded in several Engineering curricula and several are the challenges related to computer education mainly due to the high level of abstraction that this requires. In this context, different teaching methodologies are necessary. With the intention of sharing methodologies that facilitate the teaching-learning process of Vectors and Matrices, content with great difficulty, this article reports the experience of a teaching approach aided by egg boxes. The results of this experiment show that the students had a good perception about the approach, evaluating as a positive differential for learning.
\end{abstract}

Resumo. A disciplina de programação está inserida em diversos currículos de Engenharias e vários são os desafios relacionados ao ensino de computação principalmente pelo elevado nível de abstração que esta exige. Neste contexto, diferentes metodologias de ensino se fazem necessárias. Com a intenção de compartilhar metodologias que facilitam o processo de ensino-aprendizagem de Vetores e Matrizes, conteúdo com grande grau de dificuldade, este artigo relata a experiência de uma abordagem de ensino auxiliada por caixas de ovos. Os resultados dessa experiência mostram que os estudantes tiveram uma boa percepção sobre a abordagem, avaliando como um diferencial positivo para o aprendizado.

\section{Introdução}

O ensino de Programação na educação básica e ensino médio tem sido um assunto bastante discutido recentemente. No ensino superior, na maioria das grades curriculares dos cursos de graduação em Engenharias, o ensino de disciplinas de Algoritmos e Programação é obrigatório segundo as Diretrizes Curriculares Nacionais dos cursos de Engenharia ${ }^{1}$. Todas essas iniciativas de inserir Programação nos diferentes níveis de ensino, e em diversas áreas do conhecimento impulsionam a discussão sobre as metodologias de ensino. Em vários trabalhos sobre aprendizagem de programação, é

\footnotetext{
${ }^{1} \mathrm{http} / /$ portal.mec.gov.br/cne/arquivos/pdf/CES1362.pdf
} 
possível encontrar exemplos relacionados à dificuldade de lidar com ambientes e linguagens de programação [Junior e França 2017] [Castro et al. 2017]. Tais dificuldades não são encontradas apenas em turmas de Engenharias, onde os índices de evasão nas disciplinas correlatas à computação são altos, segundo INEP (Instituto Nacional de Estudos e Pesquisas Educacionais, Sinopses do ensino superior) ${ }^{2}$. Os últimos índices publicados pelas universidades quanto à evasão de estudantes nos cursos de computação contribuem com a discussão sobre os possíveis fatores que levam a esses índices. Em outubro de 2016, a USP, uma das mais conceituadas universidades do país, divulgou que mais de $20 \%$ dos (as) estudantes desistem de seus cursos, sendo os relacionados ao Instituto de Ciências e Matemática e Computação de São Carlos dentre os cursos de maior média de evasão chegando a $48 \%{ }^{3}$. Alguns estudos indicam que são variados os motivos, passando por base matemática fraca, falta de conhecimento prévio sobre o real objetivo do curso, currículos longos, critérios impróprios de avaliação do desempenho discente, entre outros [Cavalcante e Embiruçu 2013]. Porém, outro fator também apontado pelos estudos indica a não adequação da metodologia docente aos estudantes e uma falta de integração do estudante com os ambientes sócio ambientais universitários levam a um consequente nível de comprometimento afetado [Medeiros e Melo 2011]. Giraffa e Mora (2013) revelam em seu estudo que estudantes que alegavam a metodologia dos professores como um fator de desistência, demonstraram em testes específicos ter pouca dificuldade com lógica e algoritmo.

Apesar de a metodologia tradicional ainda prevalecer, tendo o professor como mero transmissor de informação e de soluções de problemas, a busca por metodologias que facilitem o aprendizado tem crescido [da Silva et al. 2015], [Sousa et al. 2010]. Dentre essas metodologias, questiona-se sobre o uso ou não da própria tecnologia como ferramenta de ensino, pois alguns movimentos defendem o afastamento do computador no ensino de Computação como o "Computer Science Unplugged" (computação "desplugada") idealizado por Bell, Witten e Fellows (2011). Essa metodologia visa o uso de atividades lúdicas, onde a aprendizagem de princípios fundamentais de computação como algoritmos e estrutura de dados acontece sem a necessidade do estudante interagir com softwares. Desta forma, "todo método ou estratégia que promova o envolvimento e a participação ativa do aluno no processo de desenvolvimento do conhecimento contribui para formar ambientes ativos de aprendizagem" [Barbosa e Moura, 2013 p. 57]. Atividades mais lúdicas podem contribuir com uma aprendizagem mais adequada a estudantes mais dinâmicos, mais ativos, como a própria proposta da metodologia ativa tende a motivar, fazendo com que estudantes façam atividades que vão além de ouvir o professor e tomar notas [Bonwell e Eison, 1991], [Barbosa e Moura, 2013]. Várias estratégias de estudo sugerem a diversificação de atividades para promover a aprendizagem mais significativa, fazendo com que os estudantes se mantenham realizando algo e pensando sobre o que estão fazendo, estimulando a habilidade de argumentação [Bonwell e Eison, 1991]; promovendo a autonomia [Berbel, 2011] e desenvolvendo a aprendizagem significativa [Rogers, 1969], [Weibell, 2011], [Moreira, 2011]. Com base nessa reflexão, o presente

\footnotetext{
${ }^{2}$ www.inep.gov.br

${ }^{3}$ http://www.jornaldocampus.usp.br/index.php/2016/10/basic-2/
} 
artigo busca compartilhar e analisar uma experiência no ensino de Vetores e Matrizes em uma disciplina de Computação em cursos de graduação em Engenharia Elétrica, Mecânica de Controle e Automação e Licenciatura em Física. A experiência diz respeito ao ensino específico de Vetores e Matrizes, utilizando caixas de ovos como material didático no apoio à redução da abstração lógica exigida, por ser um dos conteúdos em que os estudantes declaram maior dificuldade pelo alto nível de abstração que exige.

Na Seção 2 são apresentados trabalhos correlatos que também estão inspirados em metodologias ativas no ensino de computação. Na Seção 3 a experiência é compartilhada, incluindo a descrição do perfil de estudante e como foi utilizado em sala de aula. Na Seção 4 resultados e discussões são apresentados por meio das respostas dos estudantes a um questionário de avaliação da metodologia e na Seção 5, as conclusões sobre este trabalho são delineadas.

\section{Trabalhos correlatos}

O uso de recursos lúdicos e jogos (digitais ou não) vem ganhando cada vez mais espaço em diversas disciplinas dos cursos da área de Computação. Estes recursos são usados para oferecer uma experiência mais prática tanto em disciplinas mais teóricas, como os exemplos desta seção, quanto para disciplinas com conteúdo mais abstrato [Nunes e Parreira Júnior 2015] [Vahldick et al. 2015].

Uma estratégia para a disciplina de Gerenciamento de Projetos foi proposta por Schoeffel e Wazlawick (2016). A atividade vivencial: Mão na Massa foi criada como ferramenta complementar a outras abordagens para o ensino de Gerenciamento de Projetos com foco nos conceitos do $\mathrm{PMBOK}^{4}$. A atividade foi realizada em duas turmas distintas de um curso de especialização, com 35 estudantes no total. Os resultados da realização da atividade indicam que houve, segundo os próprios estudantes, uma significativa contribuição para a aprendizagem, nos três níveis de aprendizagem (conhecimento, entendimento e aplicação). Outro exemplo, também para a disciplina de Gerenciamento de Projetos, é o jogo MEGA GP [Tomisaki et al. 2016], que assim como o trabalho citado anteriormente, não utiliza recursos computacionais para realização da atividade. O MEGA GP visa à fixação de conceitos do PMBOK e do CMMI-DEV ${ }^{5}$ de maneira lúdica e motivadora. Conforme os resultados do questionário de satisfação aplicado, os estudantes ficaram satisfeitos com o uso deste recurso didático. Além disto, para a maior parte do conteúdo houve um desempenho melhor para a turma que fez uso do MEGA GP. Figueiredo e Santos (2016) apresentam um jogo de cartas de estratégia para ensinar História da Computação em diversos níveis de ensino. O jogo é composto de 60 cartas divididas em três categorias: personalidades, instituições e eventos. Além disto, cada carta pertence a uma área de conhecimento: hardware, software, teoria computacional e matemática, computação e sociedade, gestão de dados. Embora o trabalho citado não descreva a utilização do jogo em sala de aula, os autores acreditam

\footnotetext{
4 PMI. (2013). Project Management Body of Knowledge - PMBOK Newton Square. Pennsylvania: Project Management Institute. 5 ed.

5 SEI. (2010). CMMI for Development, version 1.3. Carnegie Mellon University, Software Engineering Institute (SEI).
} 
que sua dinâmica estratégica estimula o jogador na retenção dos conteúdos das cartas e a ludicidade do jogo incentiva o seu envolvimento com a disciplina.

\section{Descrição da Experiência de uso das caixas de ovos em sala de aula}

O ensino de vetores e matrizes com as caixas de ovos foi realizado em uma turma de Computação com estudantes que já haviam cursado esta disciplina, mas que haviam reprovado. Os estudantes são de cursos de graduação não relacionados à área de computação, como Licenciatura em Física, Engenharia Elétrica, Engenharia Mecânica e Engenharia de Controle e Automação. A turma era composta de 16 estudantes dos diversos períodos dos seus cursos, em média entre o $3^{\circ}$ e $4^{\circ}$ período. O material com as caixas de ovos foi utilizado primeiro no apoio ao ensino de Vetores. Os vetores foram representados em sequências horizontais de várias posições para os ovos. Para caracterizar o nome de cada posição do vetor, foram coladas pequenas tiras com os números, neste caso de 0 a 4 . Para representar o conteúdo de cada posição do vetor foram utilizados pequenos quadrados com números inteiros escritos. Por fim, para o uso na dinâmica de execução do laço que percorre o vetor, foi utilizado um post-it no formato de seta, com o nome da variável $i$ escrita.

A aula sobre vetores foi primeiramente ministrada com a metodologia expositiva com uso de quadro e slides. Após fazerem exercícios, a principal dificuldade relatada pelos estudantes foi com relação à visualização de como o laço for estava relacionado com as posições do vetor. Assim, na segunda aula, foi feita uma revisão da utilização do laço for com a estrutura de vetor. A docente escreveu no quadro o trecho de código referente ao for e reuniu a turma ao redor de uma mesa próxima ao quadro, com o vetor de caixa de ovo exposto. Após, seguiu as seguintes etapas:

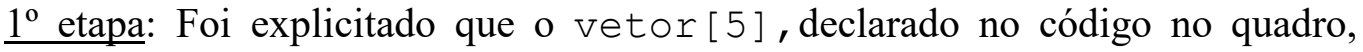
refere-se ao vetor na mesa, contando a quantidade de posições. Essa é outra dificuldade expressa pelos estudantes na aula expositiva, a confusão entre a quantidade de posições e a contagem do laço for, por ser definido que na linguagem $C$, a primeira posição do vetor é obrigatoriamente o 0 . O nome da posição impresso na caixa de ovos auxiliou nesta abstração.
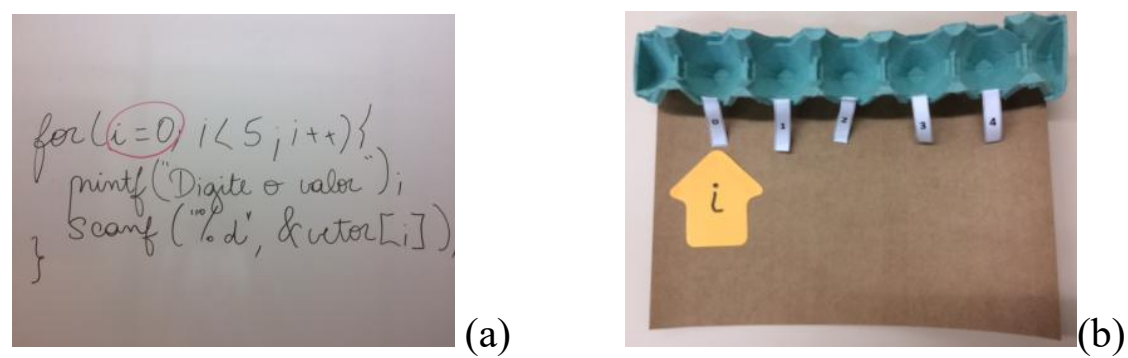

Figura 1. Atribuição do valor 0 à variável $i$

$2^{\circ}$ etapa: A docente então marca no quadro que a primeira instrução do for é executada $i=0$, a atribuição do valor zero à variável de controle $i$ (Figura 1(a)). Neste momento, o post-it no formato de seta com o nome da variável de controle $i$, passa a apontar para a posição 0 do vetor (Figura 1(b)). Esta dinâmica explicita que o i está manipulando apenas a posição do vetor e que seu conteúdo do vetor [0] ainda não foi manipulado. É importante salientar que não é correto afirmar que a posição está 
vazia, pois é sabido que a memória pode conter valores aleatórios enquanto não é diretamente manipulada por meio das variáveis. Mesmo assim, didaticamente, a posição parece vazia pela posição do "ovo" não conter nada ainda.

$3^{0}$ etapa: O próximo passo é salientar no código do quadro que a condição do laço será executada. Assim, a docente sublinha a condição i<5 (Figura 2(a)) e pergunta aos estudantes se $i$ (que neste momento possui o valor 0 ) é menor que 5, então os estudantes responderam que sim, e as instruções de dentro do laço serão executadas. Neste caso, a analogia da execução da instrução "printf” foi escrever um valor inteiro em um papel (no exemplo utilizaram o número 8) e a analogia da execução da instrução "scanf" foi colocar o papel dentro da posição do vetor (salientando o significado a instrução \&vetor [0 ] ). Essa atribuição é mostrada na Figura 2(b).

Esta dinâmica ajuda a diferenciar o valor da variável de controle do conteúdo da posição apontada pela variável de controle, e que o laço manipula $o$ i e não os conteúdos.
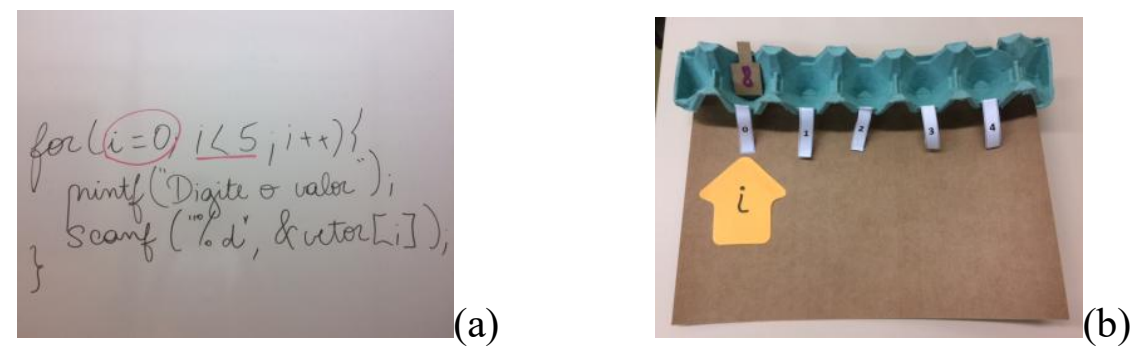

Figura 2. Atribuição do valor 9 à posição vetor [0]

4 etapa: A próxima etapa é salientar no código do quadro que o próximo passo a ser executado no laço é o incremento fazendo um retângulo em torno da instrução i++ (Figura 3(a)). Com o incremento, neste momento a variável de controle i passa a possuir o valor 1 , assim o incremento no vetor de ovos também acontece como mostra a Figura 3(b) e lembrando que a condição de $i<5$ continua sendo verdadeira neste caso, então a execução das instruções dentro do laço será efetuada.

Neste momento a abstração do funcionamento da variável de controle i já está mais concreta e os estudantes já lidam com naturalidade com a analogia da instrução printf e scanf, em que eles escrevem novamente outro valor em um papel (neste exemplo está o inteiro 30) e colocam dentro da posição do vetor [1] (Figura 3(c)).
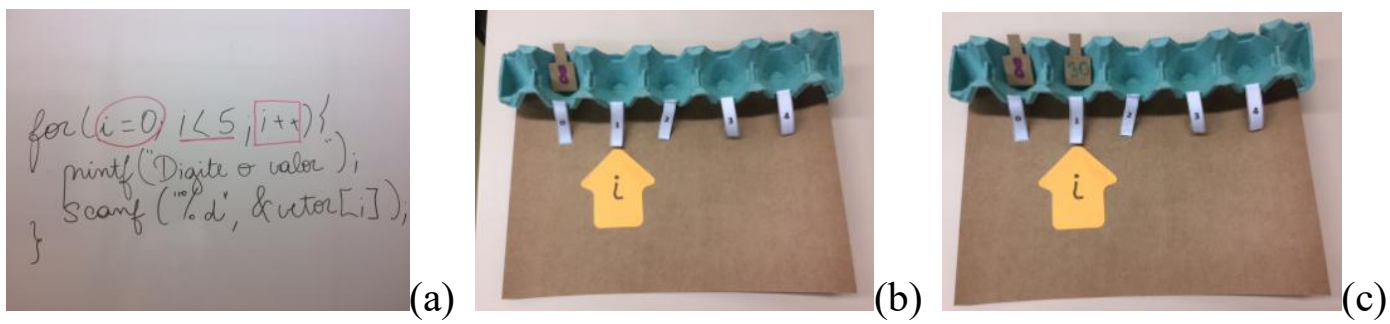

Figura 3. Execução do incremento da variável i e atribuição de valor

Após a $4^{\mathrm{a}}$ etapa, a varredura e preenchimento das outras posições tornaram-se apenas a repetição desses passos, guiado pela execução do laço for. Após o vetor estar 
completamente preenchido com os valores, foi feito um exercício semelhante para a leitura de todos os valores, bem como, exercícios que varrem o vetor de forma decrescente para que todas as oportunidades de manipulação de vetor ficassem mais concretas com o uso do vetor de caixa de ovo.

5 etapa: Após a docente fazer uso do quadro e do vetor em caixa de ovo, com todos os estudantes em grupo, foi proposta a seguinte atividade: os estudantes foram divididos em grupo e cada grupo recebeu um "kit" com os mesmos recursos utilizados pela docente contendo: um vetor de caixa de ovo, 5 papéis para escrever os valores do vetor e um post-it para o controle da variável $i$. Os estudantes deveriam utilizar o kit para resolver a lista de exercícios sobre vetores.

Em um primeiro momento, os estudantes ficaram um pouco resistentes para utilizar o kit, dizendo que não haveria necessidade porque já haviam entendido de forma concreta como manipular o vetor. Mesmo assim, foi possível observar que em alguns momentos, recorreram sim ao uso das caixas de ovos como pode ser visto na Figura 4.
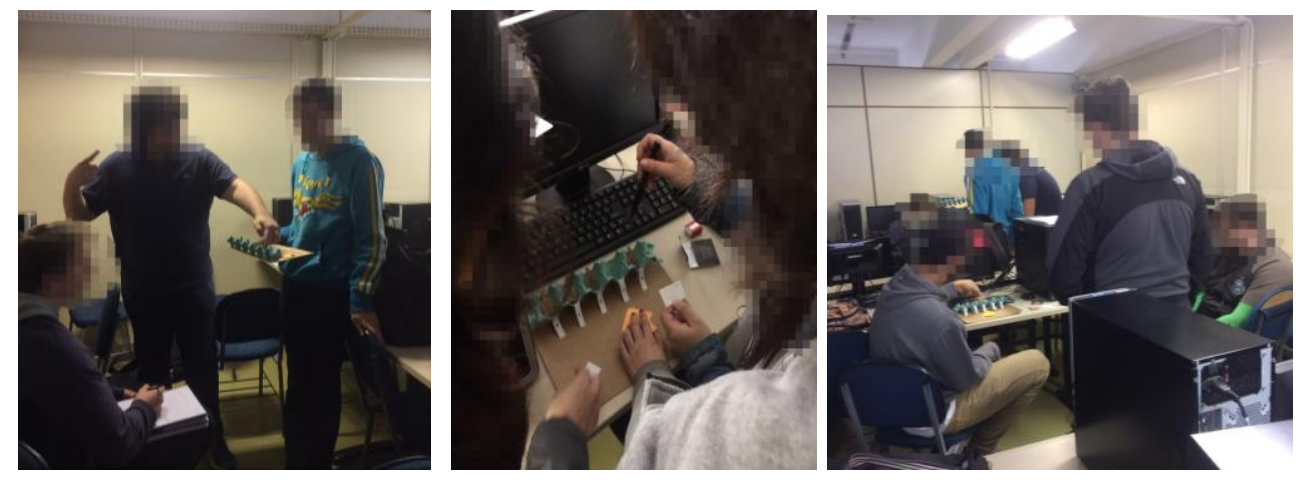

Figura 4. Estudantes utilizando os kits para resolver exercícios em grupo

Metodologia semelhante foi utilizada para o ensino de Matrizes, com a diferença que a primeira aula sobre Matrizes já foi utilizando o kit das caixas de ovos. Na Figura 5 pode ser visualizada a matriz representada com caixa de ovo. As caixas de ovos utilizadas são bastante grandes, e como a matriz para a dinâmica não precisa ser tão grande, foram cortadas matrizes quadradas de 3 linhas por 3 colunas. Dependendo da intencionalidade da aula, as matrizes podem ser não quadradas, sendo opção total do professor. Assim como no vetor, foram coladas tiras com os nomes das posições, nas matrizes as tiras possuíam um detalhe para demarcar o que estava sendo demarcado como linha (variável i) e como coluna (variável $\mathbf{j}$ ). Para uma identificação rápida e visual, foram utilizadas linhas horizontais juntamente com os nomes das posições de linha (controlas pela variável i) e linhas verticais juntamente com os nomes das posições de coluna (controladas pela variável j). Também foram utilizados 2 post-it para a marcação das variáveis $\mathbf{i}$ e $\mathbf{j}$ na dinâmica. Primeiramente a docente trouxe uma caixa de ovos para representar uma matriz quadrada (Figura 5), 2 post-its para as variáveis de controle $i$ e $j$, e papéis para colocar dentro das posições da matriz. A dinâmica também consistiu em escrever no quadro o trecho de código que percorre a matriz para atribuir valores e executar utilizando a caixa de ovos. Foram exploradas dinâmicas para preencher a matriz com valores, multiplicar matrizes e soma. Logo em seguida os grupos receberam kits para auxiliar a resolver os exercícios que envolviam 
Matrizes. Após a execução das atividades com vetores e matrizes, os estudantes foram convidados a voluntariamente responder a um questionário de avaliação sobre sua experiência de aprendizado com as caixas de ovos. Os resultados são mostrados e discutidos a seguir.

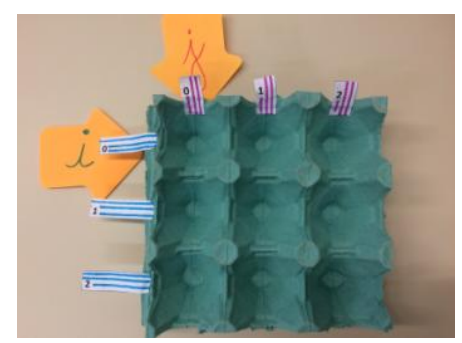

Figura 5. Matriz representada com caixa de ovo

\section{Resultados e Discussões}

Ao questionário sobre a experiência com a caixa de ovos no aprendizado de vetores, responderam 11 estudantes, visto que a resposta ao questionário era facultativa aos estudantes. O gráfico da Figura 6 mostra as respostas para a pergunta "Como você avalia a diferença que fez no seu aprendizado a explicação da docente sobre vetores com a caixa de ovos?". A maioria dos estudantes (64\%) respondeu que ajudou muito por diminuir a abstração. Ainda assim, alguns estudantes responderam que fez pouca ou nenhuma diferença, cujo julgamento pode ser, em parte, devido à resistência que alguns estudantes demonstram quando são requisitados a utilizar uma ferramenta diferente do computador. Durante a proposta da atividade, alguns demonstraram resistência por parecer ser um material que lembrava ensino fundamental ou médio.
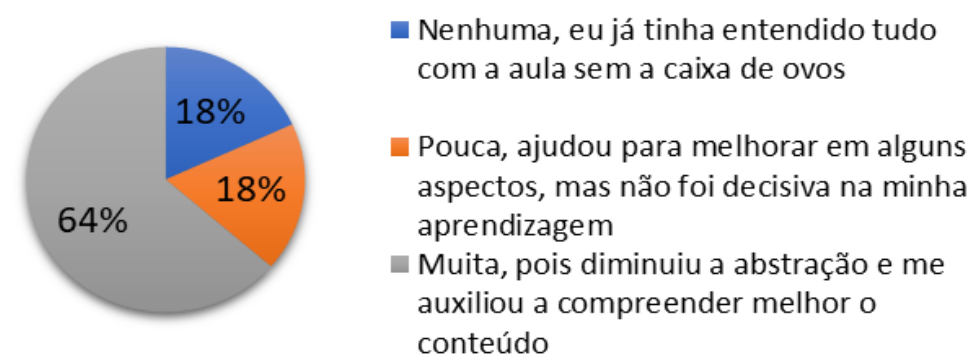

Figura 6. Respostas à pergunta sobre o aprendizado

O gráfico da Figura 7 mostra as respostas para a pergunta "Como você avalia a diferença que fez o vetor em caixa de ovos para o seu aprendizado sobre compreender a manipulação do incrementador dos índices (i)"? A maioria dos estudantes (73\%) respondeu que o vetor em caixa de ovos ajudou muito no seu aprendizado por auxiliar a ver como o $i$ funciona dentro do laço. Essas respostas dão fortes indícios de que a metodologia de fato auxilia na compreensão da manipulação do laço, pois mesmo quem respondeu que fez pouca diferença, ainda afirma que ajudou a melhorar a compreensão devido á manipulação concreta da variável i com o post-it. 


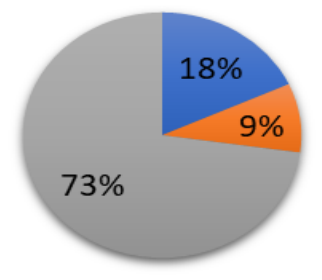
- Nenhuma, eu já tinha entendido com a aula sem a caixa de ovos
- Pouca, ajudou a melhorar porque pude manipular o i com as mãos
Muita, pois consegui ver como o $\mathrm{i}$ funciona dentro do laço

Figura 7. Respostas à pergunta sobre a variável de controle $\mathrm{i}$

O gráfico da Figura 8 mostra as respostas para a pergunta "Como você avalia a diferença que fez no seu aprendizado a explicação da docente sobre compreender o que deve ficar dentro e fora do laço ao percorrer um vetor?". Observando todos os gráficos, este foi o que obteve maior porcentagem de resposta quanto à metodologia ter feito muita diferença ( $82 \%$ das respostas). Esse é um fator bastante positivo, por mostrar que o entendimento da estrutura do laço for não é trivial por exigir uma abstração de identificar em que momento exatamente cada uma das 3 instruções é executada. Com os destaques no código escrito no quadro, juntamente com a manipulação do vetor, auxilia a compreender. Quanto às outras respostas, novamente associamos à resistência com $o$ uso do artefato.
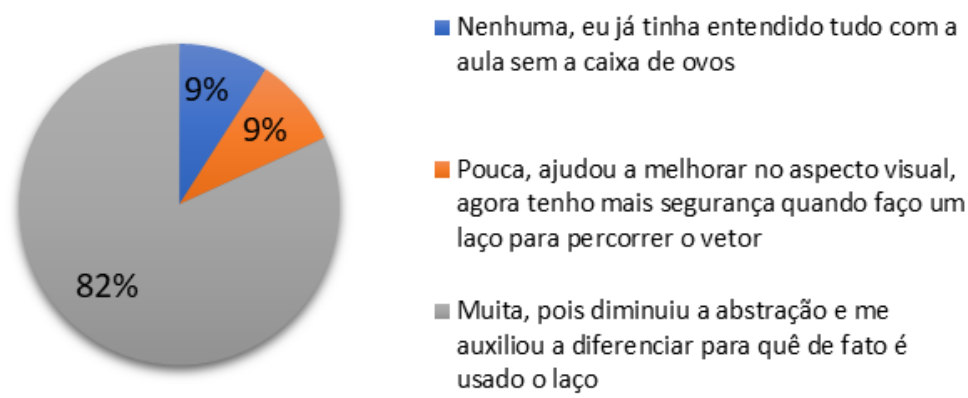

Figura 8. Respostas à pergunta sobre o laço que percorre o vetor

Os estudantes também foram convidados a voluntariamente avaliar seu aprendizado com as matrizes em caixas de ovos. Porém, nesta avaliação, apenas 4 estudantes responderam ao questionário. Para as perguntas "Como você avalia a diferença que fez no seu aprendizado a explicação da docente sobre matrizes com a caixa de ovos"? e "Como você avalia a diferença que fez no seu aprendizado a explicação com caixa de ovos para compreender o for aninhado?", as respostas refletiram 50\% para "Muita pois diminuiu a abstração" e 50\% para "Pouca, ajudou a melhorar no aspecto visual, assim adquiri mais segurança quando faço um laço aninhado para manipular matrizes" e nenhum aluno respondeu que não fez diferença alguma.

Para as matrizes salientamos as respostas à pergunta " $\mathrm{Na}$ sua opinião, você acredita que foi melhor aprender os conceitos primeiro com a caixa de ovos do que com slides apenas?", mostrado na Figura 9. Para esta pergunta, apesar de poucas respostas, a maioria $(75 \%)$ dos estudantes afirma que aprender com os artefatos foi melhor do que apenas com slides, metodologia predominante em aulas expositivas. 

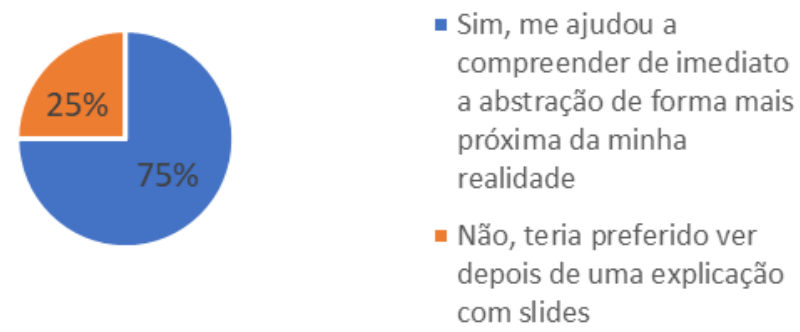

Figura 9. Resposta sobre o aprendizado de matrizes

Embora a aprendizagem significativa tenha ocorrido em aspectos como manipulação de laço, da variável de controle as relações disso tudo com os conteúdos das estruturas de dados, é interessante salientar a surpresa dos estudantes ao verem os kits de caixas de ovos bem como a resistência inicial em utilizarem os mesmos. Mesmo quando os estudantes confirmam que a caracterização visual das caixas de ovos em vetores e matrizes, juntamente com os códigos escritos no quadro fizeram com que o conteúdo passasse do nível abstrato para concreto, ainda assim, nota-se que a estratégia metodológica utilizada pela docente não foi de todo compreendida por eles como facilitadora da aprendizagem.

\section{Conclusões}

Historicamente, na disciplina de Computação em que a metodologia exposta neste artigo foi aplicada, o ensino de vetores e matrizes é um dos mais temidos conteúdos abordados. A partir dessa constatação, foi desenvolvida a metodologia que fez uso de um artefato sustentável construído artesanalmente com caixas de ovos, que de fato, resultou positivamente com relação à melhora na retenção de estudantes na disciplina. Apenas 1 aluno desta turma reprovou, e, apesar de não ser possível apenas com uma aplicação da metodologia afirmar que esse foi o fator determinante para o sucesso dos estudantes. Assim, considera-se que o método foi válido no sentido de concretizar conceitos abstratos para a aprendizagem significativa sobre algoritmos pelos estudantes.

\section{Referências}

Barbosa, E.F., Moura, D.G. (2013) Metodologias ativas de aprendizagem na educação profissional e tecnológica. Boletim Técnico SENAC. Rio de Janeiro, v.39, n.2, p.4867, maio-ago.

Bell, T.; Witten, I.H., Fellows, M. (2011) Computer Science Unplugged: ensinando Ciência da Computação sem o uso do computador. Tradução coordenada por Luciano Porto Barreto. p.3- 45.

Berbel, N. (2011) As metodologias ativas e a promoção da autonomia dos estudantes. Semina: Ciências Sociais e Humanas, Londrina, v. 32, n. 1, p. 25-40

Bonwell, C. C., Eison, J.A. (1991) Active Learning. Creating Excitement in the Classroom. ASHE-ERIC Higher Education Report. 121 p.

Castro, R.M.C., Siqueira, S.W.M., Almeida, D.N.; Nascimento, F.C. (2017) Agility Scrum - Um jogo para Ensino da Metodologia SCRUM. 25 WEI - Workshop sobre Educação em Computação. São Paulo. p.2217-226 
Cavalcante, F. P.L., Embiruçu, M. S. (2013) Aprendizado com base em problemas: Como entusiasmar os estudantes e reduzir a evasão nos cursos de graduação em engenharia. XLI Congresso Brasileiro de Ensino em Engenharia, v. 2013.

Da Silva, S.F., Barbosa, A.F., de Souza, A.A., da Silva, E.G., Silva, M.L., Neto, S.R.S., dos Santos, W.O. (2015) Relato de Experiência de Ensino de Computação no Ensino Fundamental em Estágio Supervisionado da Universidade de Pernambuco no Campus Garanhuns. In: XXIII Workshop sobre Educação em Computação.

Figueiredo, K. da S., Santos, J. C. O. (2016) Computasseia: Um Jogo para o ensino de História da Computação. In: XXIV Workshop sobre Educação em Informática (WEI 2016). p. $2026-2035$.

Giraffa, Lucia, Mora, Michael. (2013) Evasão na Disciplina de Algoritmo e Programação: Um Estudo a partir dos Fatores Intervenientes na Perspectiva do Aluno. Conferência Latino-americana Sobre El Abandono En La Edcación Superior - III.

Junior, S.M.S., França, S.A. (2017) Programação para todos: Análise Comparativa de Ferramentas Utilizadas no Ensino de Programação. 25 WEI - Workshop sobre Educação em Computação. São Paulo.

Medeiros, S.M.O., Melo, J.C.B. (2011) Metodologias para o Ensino de Ciência da Computação na Educação Básica. XI Jornada de Ensino, Pesquisa e Extensão JEPEX 2011 - UFRPE. Recife

Moreira, M.A. (2011) Aprendizagem significativa: a teoria e textos complementares. São Paulo: Livraria Editora da Física.

Nunes, I. F. e Parreira Júnior, P. A. (2015) RPG4Sorting - Um Jogo Educacional para Auxílio ao Ensino de Métodos de Ordenação, In: XXIII Workshop sobre Educação em Informática (WEI 2015).

Rogers, C. (1969) Freedom to learn: a view of what education might become. Columbus, OH: Charles E. Merrill Pub. Co. 358p.

Schoeffel, P., Wazlawick, R.S. (2016) Mão na Massa: Dinâmica Vivencial para Apoio ao Ensino de Gerenciamento de Projetos de Software. In: XXIV Workshop sobre Educação em Informática (WEI 2016) p. 2215 - 2224.

Sousa, R. V., Barreto, L. P., Andrade, A., Abdalla, D. (2010) Ensinando e aprendendo conceitos sobre ciência da computação sem o uso do computador: Computação Unplugged! Congresso Brasileiro de Informática na Educação. Volume 1, No 1. p.5.

Tomisaki, S. M. M., Souza, A. D.; Seabra, R. D. (2016) MEGA GP: Aplicando a Gamificação no Ensino de Gerência de Projetos. In: XXIV Workshop sobre Educação em Informática (WEI 2016) p. 2225 - 2234.

Vahldick, A., Mendes, A. J., Marcelino, M. J., Hogenn, M., Schoeffel, P. (2015) "Testando a Diversão em um Jogo Sério para o Aprendizado Introdutório de Programação”, In: XXIII Workshop sobre Educação em Informática (WEI 2015).

Weibell, C. J. (2011). Principles of learning: 7 principles to guide personalized, studentcentered learning in the technology-enhanced, blended learning environment. 